\title{
Basilar Bifurcation Aneurysms
}

\author{
Atos Alves de SousA \\ Faculdade de Ciências Médicas de Minas Gerais, Santa Casa de Belo Horizonte, \\ Minas Gerais, Brazil
}

\begin{abstract}
Direct operative management of basilar bifurcation aneurysms is one of the most challenging procedures in the realm of vascular neurosurgery, as these lesions are deeply situated in the interpeduncular region and maintain an intimate relationship with important anatomical structures. Direct clipping of aneurysms generally represents the gold standard of surgical treatment, as it allows exclusion of the aneurysmal sac from the circulation, evacuation of aneurysmal contents for decompression, and preservation of efferent flow. The author describes his experience with 123 operated patients of basilar bifurcation aneurysms from January 1977 till December 1995. In these 123 consecutive surgeries the results were $85 \%$ good outcome, $8.1 \%$ morbidity, and $6.5 \%$ mortality. In the first years of this series the pterional or subtemporal approaches were used, depending on the level of the basilar bifurcation, the exact origin of the sac, its projection and size. From 1987 on all patients were operated by a modified pterional approach described initially by Sano as temporopolar approach.
\end{abstract}

Key words: cerebral aneurysms, basilar artery, temporopolar approach

\section{Introduction}

Posterior fossa aneurysms account for $5 \%$ to $15 \%$ of all intracranial aneurysms. Among infratentorial aneurysms, those of the basilar bifurcation are the most common (representing around $50 \%$ ) and may account for $5 \%$ to $8 \%$ of all intracranial aneurysms. ${ }^{5,151}$

The development of the surgical management of basilar artery aneurysms parallels the career of Drake. In 1961, Drake ${ }^{51}$ reported for the first time his experience with four cases of direct surgical management of ruptured basilar tip aneurysms. Although two of those first four patients died postoperatively, the two survivors had astonishing functional recoveries.

The initial unacceptable morbidity and mortality obtained by many authors ${ }^{2,10)}$ have progressively improved, although they still remain much higher than those for the aneurysms of the anterior circulation. A more detailed anatomical knowledge of the region, the importance of preserving the perforating vessels, the development of microsurgical techniques, improved aneurysm clips, anesthetic techniques, intraoperative monitoring, pre- and postoperative care, and neuroradiologic diagnoses have resulted in improved clinical outcome and in lower rates of morbidity and mortality as well.
The direction of the fundus is an important factor at this location of aneurysm, both with regard to the technical difficulty encountered at the operation and to the ultimate prognosis. ${ }^{15}$ The fundus can project in three primary directions: superiorly, posteriorly, and anteriorly. The perforating arteries arising from the dorsal aspect of the basilar artery and $P_{1}$ segments of the posterior cerebral arteries are usually related to the posterolateral aspects of the aneurysm sac. The most difficult lesions to approach are those aneurysms projecting posteriorly as they are closely attached to the perforating arteries thus making the aneurysm dissection extremely hard. Aneurysms that project anteriorly toward the dorsum sellae are the less difficult to approach as they are directed away from the perforating arteries. The most common projection is the superior. Such aneurysms project toward the posterior diencephalon and the perforating arteries tend to course away from the neck.

\section{Materials and Methods}

In the present series the first patients were treated by a subtemporal approach as described by Drake ${ }^{51}$ or by a pterional approach as proposed by Yasargil $^{17.18)}$ depending on the level of the basilar bifurcation and the projection of the aneurysm. In the past 9 


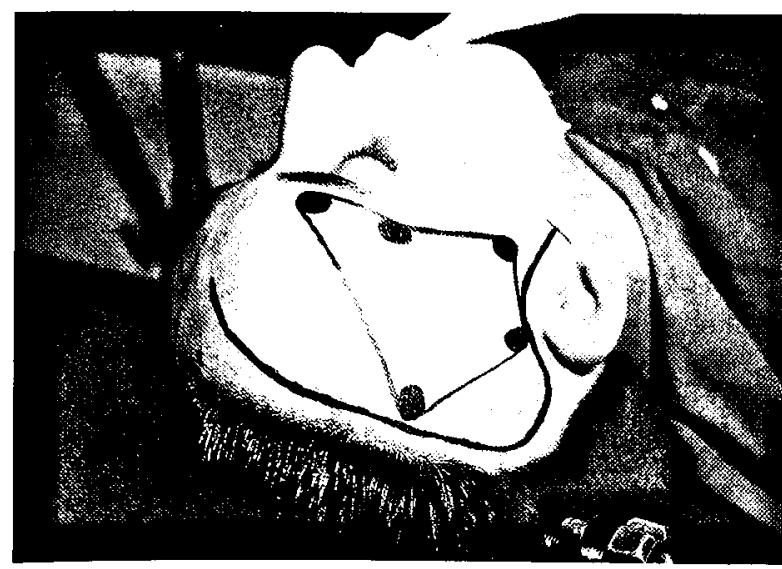

Fig. 1 Positioning of the patient, skin incision, and craniotomy.

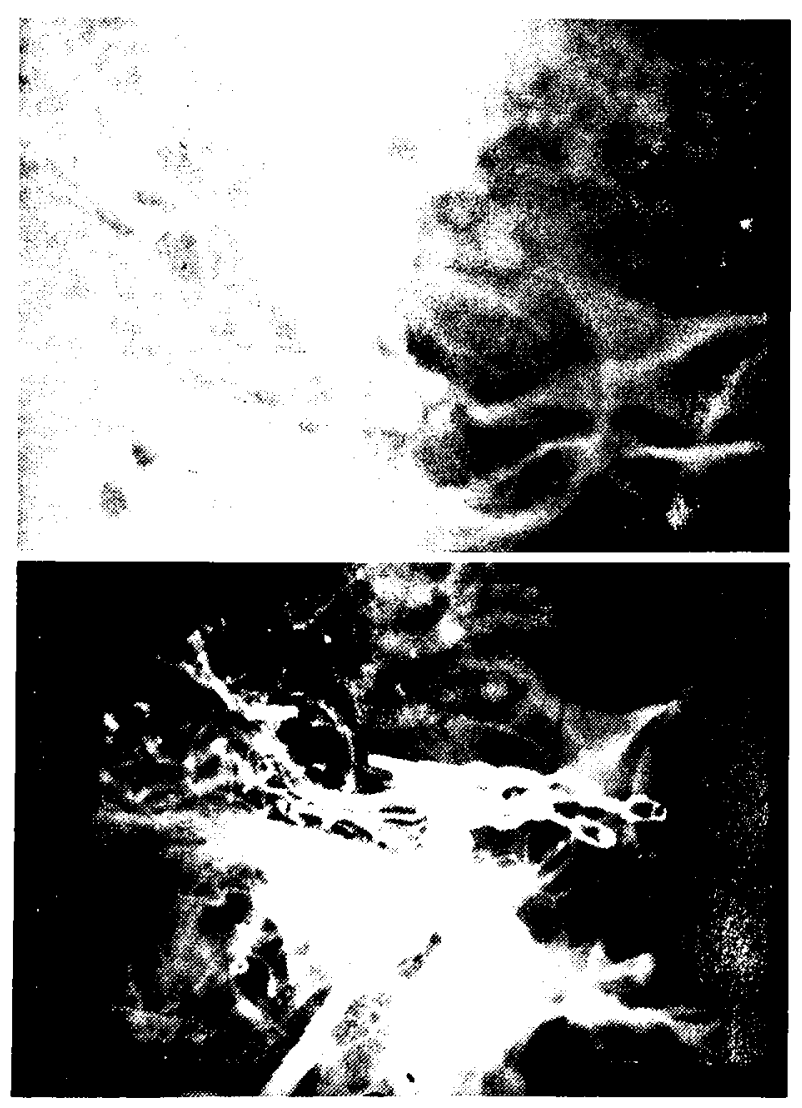

Fig. 2 Illustrative case: basilar bifurcation aneurysm. upper: Preoperative angiogram, lower: postoperative angiogram.

years all of our patients have been operated by the modified pterional approach (Fig. 1) initially described as temporopolar approach by Sano ${ }^{14)}$ and later as anterior temporal approach by Sundt, ${ }^{16)}$ or pretemporal approach by Oliveira et al. ${ }^{12)}$ This approach combines the advantages of both the subtemporal and pterional approaches providing access to the interpeduncular cistern through the posterior elevation of the temporal lobe. For giant or complex aneurysms (Fig. 2), the craniotomy can be extended to include the resection of the zygomatic $\operatorname{arch}^{11)}$ or the removal of the superior orbital rim along with the zygomatic arch. ${ }^{2,6-9,13)}$ In cases of low situated basilar bifurcation aneurysms, this approach can be associated with the resection of the posterior clinoid process or even with the transsellar-transcavernous route described by Dolenc et al. ${ }^{41}$ On some occasions it could be necessary to divide the circle of Willis at some point to provide more working space. Yasargil $^{17]}$ described the sacrifice of the posterior communicating artery, posterior cerebral artery, and anterior cerebral artery. In our series we have experience only with the sacrifice of the posterior communicating artery, and no complications related to the procedure resulted in such cases.

It must be said that all patients in this series were operated at least a week after the subarachnoid hemorrhage because that was our policy for the timing of operation of basilar artery aneurysms. This policy changed in 1996 to the surgical approach in the acute phase not only for basilar artery but also for all posterior circulation aneurysms.

\section{Results}

From January 1977 to December 1995 the author operated 1712 aneurysm patients of whom 198 were cases of posterior circulation aneurysms. In 123 patients, the aneurysm arose from the basilar bifurcation (accounting for $62.8 \%$ of vertebrobasilar, and for $7.2 \%$ of the whole series).

There were 20 additional aneurysms in the internal carotid artery, 17 in the middle cerebral artery, and 15 in the anterior communicating artery complex. In such cases of multiple aneurysms, we tried to clip all of them at the same operation. That was possible in all cases except for five aneurysms contralateral to the approach, three of which in the middle cerebral artery and two in the internal carotid artery.

Of the 123 patients, $59(48 \%)$ were males and 64 $(52 \%)$ were females. Ages ranged from 17 to 73 years, the average being 46.120 patients (97.6\%) manifested clinically by subarachnoid hemorrhage and three $(2.4 \%)$ unruptured giant aneurysms manifested by brain stem compression.

All operated patients were Hunt and Hess grades I, II, or III. We did not operate any patients in grades IV and $\mathrm{V}$ at this location. 
In 117 cases it was possible to clip the aneurysm, but in two cases the clipping was incomplete. In the remaining six patients, in whom we were not able to clip the aneurysm, it was covered with muscle and/ or nylon fabric.

In this group of 123 patients, 105 patients (85\%) had good recoveries, $10(8.1 \%)$ showed a fair result, and eight $(6.5 \%)$ died. The deaths were related to complications such as rerupture of two wrapped aneurysms, temporary clipping of the basilar artery for a long period of time in two patients, lesions of perforating arteries in one case, and vasospasm and clinical complications in three cases.

\section{Discussion}

Although in many services around the world the majority of basilar bifurcation aneurysms are nowadays treated by embolization with Guglielmi detachable coils, we still treat all aneurysms at any location surgically. The reason is that the endovascular embolization of aneurysms technique is not available in our service.

As most vascular neurosurgeons in various countries have been doing in the past few years, we have operated all basilar tip aneurysms by temporopolar approach with more or less bone resection depending mostly on the size of the aneurysm. This kind of approach allows a large operative field thus provid-

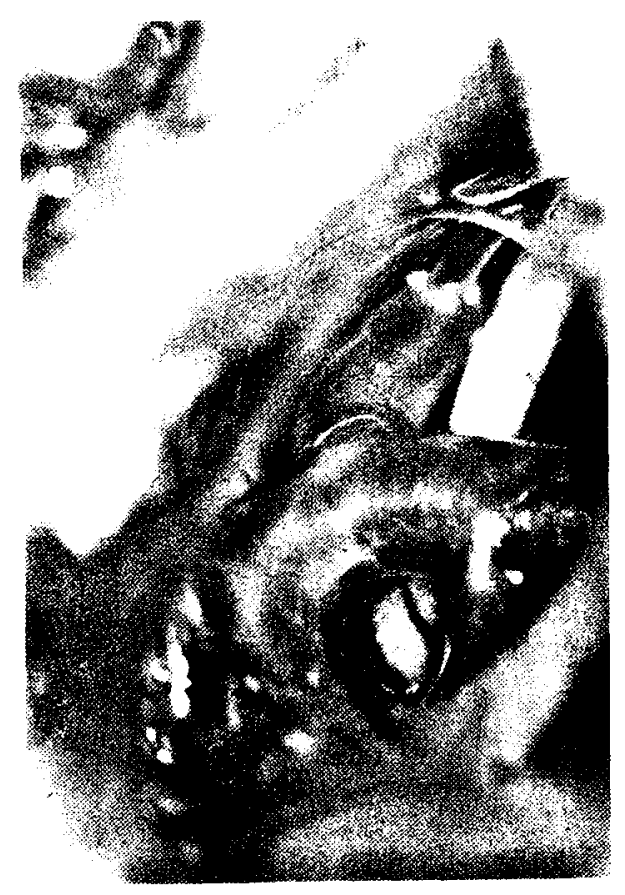

Fig. 3 Exposure of the interpeduncular cistern and basilar bifurcation. ing multiple angles of view through the pterional as well as the subtemporal routes (Fig. 3).

The obtained results are not as good as those of aneurysms in the anterior circulation. We had $14.6 \%$ of combined mortality and morbidity for patients in good grades (I-III). But as we compare our results to those found in the literature, ${ }^{5,14,15)}$ they are not any different from the average good results. Therefore we can infer that this location of aneurysms offers much more difficulties which are related to the restricted operative field of the interpeduncular region, the depth of the exposure, and the proximity to important neuro- and vascular structures. ${ }^{1)}$

\section{References}

1) Batjer $\mathrm{HH}$, Samson DS: Causes of morbidity and mortality from surgery of aneurysms of the distal basilar artery. Neurosurgery 25: 904-916, 1989

2) Chou S, Ortiz-Suarez HJ: Surgical treatment of arterial aneurysms of the vertebrobasilar circulation. J Neurosurg 4: 671-676, 1974

3) Day JD, Giannotta SL, Fukushima T: Extradural temporopolar approach to lesions of the upper basilar artery and infrachiasmatic region. J Neurosurg 81: 230235, 1994

4) Dolenc VV, Skrap M, Sustersic J, Skrbec M, Morina A: A transcavernous-transsellar approach to the basilar tip aneurysms. Br J Neurosurg 1: 251-259, 1987

5) Drake CG: Bleeding aneurysm of the basilar artery: direct surgical management in four cases. $J$ Neurosurg 18: 230-238, 1961

6) Drake CG: The treatment of aneurysms of the posterior circulation. Clin Neurosurg 26: 96-144, 1979

7) Fujitsu K, Kuwabara T: Zygomatic approach for lesions in the interpeduncular cistern. J Neurosurg 62: 340-343, 1985

8) Hakuba A, Liu S, Nishimura S: The orbitozygomatic infratemporal approach: a new surgical technique. Surg Neurol 26: 271-276, 1986

9) Ikeda K, Yamashita J, Hashimoto M, Futami K: Orbitozygomatic temporopolar approach for high basilar tip aneurysm associated with a short intracranial internal carotid artery: a new surgical approach. Neurosurgery 28: 105-110, 1991

10) Jamieson KG: Aneurysms of the vertebrobasilar system. Surgical intervention in 19 cases. J Neurosurg 28: 544-549, 1964

11) Neil-Dwyer G, Sharr M, Haskell R, Currie D, Hosseini $\mathrm{M}$ : Zygomatic-temporal approach to the basis cranii and basilar artery. Neurosurgery 23: 20-22, 1988

12) Oliveira E, Tedeschi H, Siqueira MG, Peace DA: The pretemporal approach to the interpeduncular and petroclival regions. Acta Neurochir (Wien) 136: 204211, 1995

13) Pittelli SD, Almeida GM, Nakagawa EJ, Marchese 
AT, Cabral ND: Basilar aneurysm surgery: the subtemporal approach with section of the zygomatic arch. Neurosurgery 18: 125-128, 1986

14) Sano K: Temporo-polar approach to aneurysms of the basilar artery at and around the distal bifurcation: technical note. Neurol Res 2: 261-267, 1980

15) Shiokawa Y, Saito I, Aoki N, Mizutani H: Zygomatic temporo-polar approach for basilar artery aneurysms. Neurosurgery 25: 793-797, 1989

16) Sundt TM Jr: Modified Pterional (Anterior Temporal) Approach. Surgical Techniques for Saccular and Giant Intracranial Aneurysms. Baltimore, Williams \& Wilkins, 1990, pp 213-223
17) Yasargil MG: Microneurosurgery, vol II. Stuttgart Thieme, 1984, pp 232-259

18) Yasargil MG, Antic J, Laciga R, Jain KK, Hodosch RM, Smith RD: Microsurgical pterional approach to aneurysms of the basilar bifurcation. Surg Neurol 6: 83-91, 1976

Address reprint requests to: A. A. de Sousa, M.D., Faculdade de Ciências Médicas de Minas Gerais, Santa Casa de Belo Horizonte, Rua Ceará, $567-5^{\circ}$. andar, 30.150.310 Belo Horizonte, Minas Gerais, Brazil. 